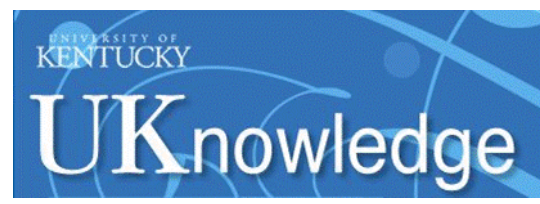

University of Kentucky

UKnowledge

9-15-1982

\title{
Physical Conditions in the Accretion Disk of V603 Aquilae
}

\author{
Gary J. Ferland \\ University of Kentucky, gary@uky.edu \\ D. L. Lambert \\ University of Texas \\ M. L. McCall \\ University of Texas \\ G. A. Shields \\ University of Texas \\ M. Slovak \\ University of Texas
}

Follow this and additional works at: https://uknowledge.uky.edu/physastron_facpub

Part of the Astrophysics and Astronomy Commons, and the Physics Commons

Right click to open a feedback form in a new tab to let us know how this document benefits you.

\section{Repository Citation}

Ferland, Gary J.; Lambert, D. L.; McCall, M. L.; Shields, G. A.; and Slovak, M., "Physical Conditions in the Accretion Disk of V603 Aquilae" (1982). Physics and Astronomy Faculty Publications. 184.

https://uknowledge.uky.edu/physastron_facpub/184

This Article is brought to you for free and open access by the Physics and Astronomy at UKnowledge. It has been accepted for inclusion in Physics and Astronomy Faculty Publications by an authorized administrator of UKnowledge. For more information, please contact UKnowledge@lsv.uky.edu. 


\section{Physical Conditions in the Accretion Disk of V603 Aquilae}

Digital Object Identifier (DOI)

http://dx.doi.org/10.1086/160298

\section{Notes/Citation Information}

Published in The Astrophysical Journal, v. 260, no. 2, p. 794-806.

(C) 1982. The American Astronomical Society. All rights reserved.

The copyright holder has granted permission for posting the article here. 


\title{
PHYSICAL CONDITIONS IN THE ACCRETION DISK OF V603 AQUILAE
}

\author{
G. J. FerLand \\ Department of Physics and Astronomy, University of Kentucky, Lexington \\ AND \\ D. L. Lambert, ${ }^{1}$ M. L. McCall, ${ }^{2}$ G. A. Shields, ${ }^{3}$ AND M. H. SlovaK ${ }^{1}$ \\ Department of Astronomy, University of Texas, Austin \\ Received 1981 December 21; accepted 1982 March 24
}

\begin{abstract}
Ultraviolet and optical spectra of the old nova V603 Aql are discussed. The UV-optical continuum is dominated by emission from the accretion disk. Emission lines from ions of $\mathrm{H}, \mathrm{He}, \mathrm{C}, \mathrm{N}$, and $\mathrm{O}$ are identified. These lines are probably formed in a circumstellar shell with radius comparable to the binary separation, density $\sim 10^{10} \mathrm{~cm}^{-3}$, and a roughly solar chemical composition. This corona is probably heated by radiation emitted by the underlying accretion disk. Photoionization calculations of the structure and emission-line spectrum of the corona are presented, and the effects of this gas on the X-ray continuum are discussed.
\end{abstract}

Subject headings: stars: accretion — stars: individual — stars: novae — ultraviolet: spectra

\section{INTRODUCTION}

This paper discusses satellite ultraviolet and groundbased optical spectrophotometric observations of the old galactic nova V603 Aql. Nova Aql 1918, which is the prototype of the "fast" class of galactic novae, is the brightest known nova remnant (Payne-Gaposchkin 1957). The material ejected in the 1918 eruption was visible as an expanding disk throughout the 1920s. Application of the nebular expansion parallax method makes this nova one of the few with a secure distance ( $d=380$ pc; McLaughlin 1960). Both the brightness of the remnant and its position as a "standard" nova make V603 Aql an attractive candidate for study with IUE. This paper presents a reconnaissance of the ultraviolet spectrum (§ II) and estimates the physical conditions in the disk ( $\S$ III) and emission-line region ( IV). An interpretation of the emission-line spectrum in terms of photoionization by the hard continuum originating in hot inner regions of the disk and boundary layer is presented in $\S \mathrm{V}$.

\section{OBSERVATIONS}

The nova V603 Aql was observed with the International Ultraviolet Explorer over the wavelength interval $1200 \AA<\lambda<3300 \AA$ (see Fig. 1). The IUE observations are summarized in Table 1. Standard image processing

\footnotetext{
${ }^{1}$ Guest observer with the International Ultraviolet Explorer satellite.

${ }^{2}$ Visiting Astronomer, Kitt Peak National Observatory, operated by the Association of Universities for Research in Astronomy, Inc. under contract with the National Science Foundation.

${ }^{3}$ Alfred P. Sloan Research Fellow.
}

was employed with the flux calibration of Bohlin and Snijders (1978). The photometric calibration is thought to be accurate to $\sim 10 \%$. The resolution is about $6 \AA$. Inspection of the vidicon images shows that the width of the spectrum perpendicular to the dispersion is entirely instrumental in origin. Therefore, the emission lines and the continuous spectrum originate in the compact central source and not the extended shell ejected in 1918.

Two optical observations were made with the IDS spectrometer on the Kitt Peak $4 \mathrm{~m}$ telescope (Fig. 2). Star and sky were measured simultaneously through adjacent diaphragms, and the background was subtracted. Data covering $3400 \AA \lesssim \lambda \lesssim 5200 \AA$ were obtained on 1979 November 24, and the interval 4700 $\AA \lesssim \lambda \lesssim 6900 \AA$ A was covered the next night. These spectra, which were measured together with a number of suitable standard stars, were corrected for atmospheric extinction and reduced to the Hayes and Latham (1975) absolute calibration of Vega. The data are believed to be accurate to $\sim 15 \%$. The resolution is about $10 \AA$.

A composite ultraviolet-optical flux curve is shown in Figure 3. The two short-wavelength $I U E$ exposures are in good agreement; the fluxes differ by $16 \%$ at $1250 \AA$ and just $1 \%$ at $1850 \AA$. A larger difference is noted for the overlap wavelengths in exposures SWP 8756 and LWR 7499; at $1950 \AA$, the fluxes differ by $33 \%$. The discrepancy surely reflects an intrinsic variation of the brightness of V603 Aql. In general, cataclysmic variables display at least two types of photometric activity; a rapid "flickering," believed to be due to small changes in the accretion stream striking the disk and forming a "hot spot" (Warner and Nather 1971), and a long term 


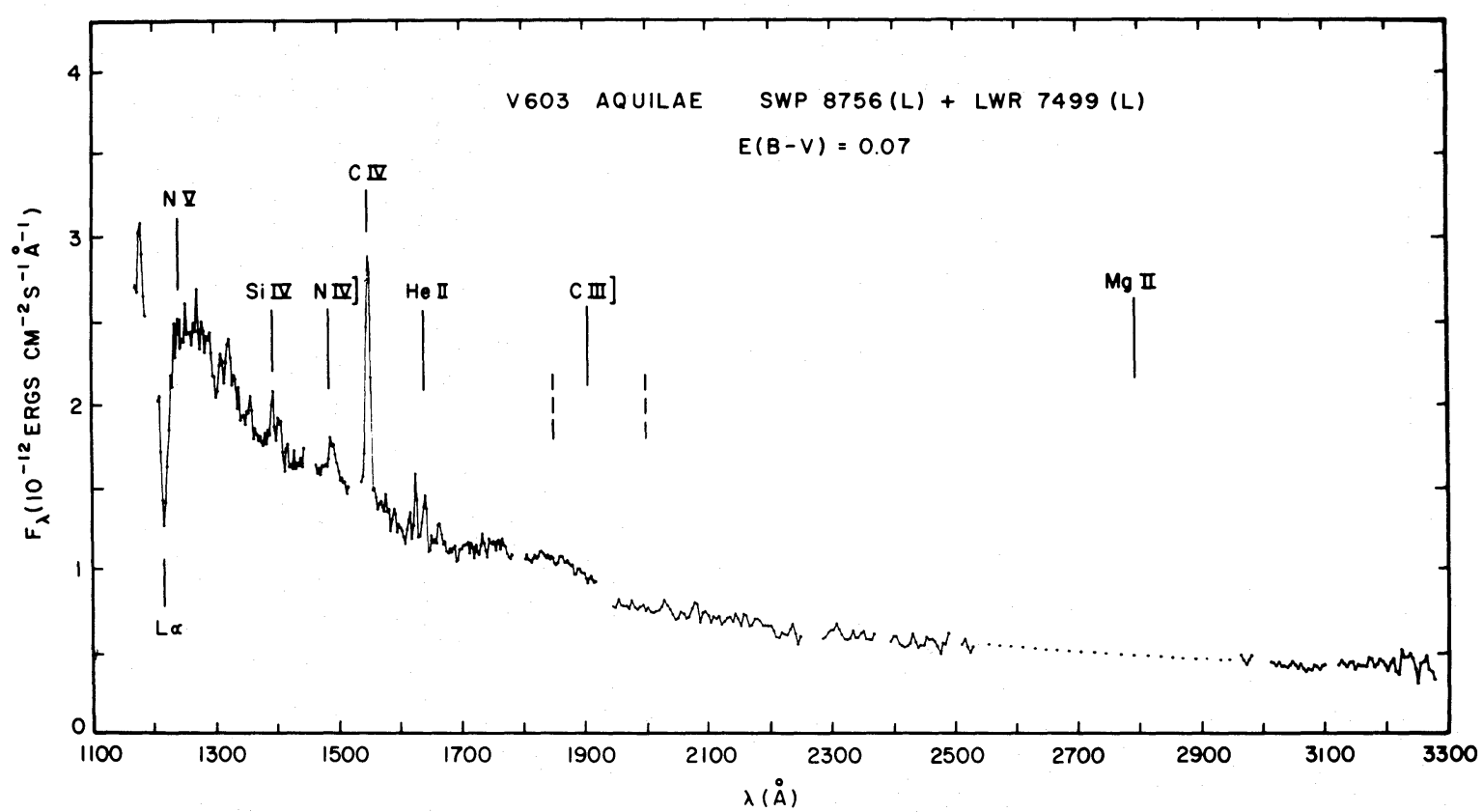

FIG. 1.-A composite $\lambda \lambda 1150-3300 \AA$ ultraviolet spectrum was obtained by combining low-resolution IUE short and long-wave spectra. The interval $\lambda \lambda 2250-2950 \AA$ was overexposed and is not shown. A color excess of $E(B-V)=0.07$ mag and the extinction curves of Nandy et al. (1976) were used to correct the spectra for interstellar reddening.

TABLE 1

SUMMARY OF IUE OBSERVATIONS ${ }^{\mathrm{a}}$

\begin{tabular}{lccc}
\hline \hline IUE Image & Dispersion & $\begin{array}{c}\text { Exposure } \\
(\mathrm{min})\end{array}$ & $\begin{array}{c}\text { JD } \\
\text { (JD 2,440,000+) }\end{array}$ \\
\hline SWP 5678 $\ldots$ & Low & 13 & 4054.375 \\
SWP 8756 $\ldots$ & Low & 13 & 4343.944 \\
LWR 7499 $\ldots$ & Low & 30 & 4343.962
\end{tabular}

aAll observations were made through the $10^{\prime \prime} \times 20^{\prime \prime}$ large aperture.

secular change (Warner 1976). Time-resolved spectrophotometry (Panek 1979) shows that variations of $\sim 0.3$ mag over time scales of $\sim 10$ minutes are common in V603 Aql. Panek (1979) also found that the optical variations were gray; if this is true for the ultraviolet, then the $\sim 0.3$ mag uncertainty will also affect the $I U E$ observations.

The moderate resolution observations of Wyckoff and Wehinger (1978) and Panek's (1979) photometry both confirm the rather flat $\left(f_{v} \sim\right.$ constant) form of the optical energy distribution. There is a $\pm 0.3 \mathrm{mag}$ scatter in the actual flux density in the continuum as measured in the three studies. Presumably, these differences reflect secular changes in the brightness of the nova. The IUE spectra are in qualitative agreement with earlier ultraviolet photometry (Gallagher and Holm 1974; see Lambert et al. 1980).
The uncertainty in the true level and shape of the continuum is exacerbated by the effects of interstellar reddening. Even a small reddening of $E_{B-V}=0.07 \mathrm{mag}$, consistent with estimates of the distance to V603 Aql, will raise the UV/optical intensity ratio by a factor of 2 (Nandy et al. 1976). Considering these uncertainties, power-law continua in the range $f_{\nu} \propto \nu^{0}-f_{\nu} \propto \nu^{0.5}$ are consistent with the observations. The continuum is clearly not that of a hot blackbody $\left(f_{\nu} \propto \nu^{2}\right)$.

Table 2 lists line intensities and upper limits as measured from the two SWP IUE spectra. Like the continuum, these lines appear to be variable (although they do not vary together; the equivalent width of the strong $\mathrm{C}$ IV emission line is not constant). Mean intensities are listed in the last column, and Table 3 lists the mean intensities of all detected ultraviolet and optical emission lines. The two entries for $\mathrm{H} \beta$ correspond to independent measurements from the blue and red KPNO data. The last column of Table 3 gives the line intensities after a reddening correction for $E_{B-V}=0.07 \mathrm{mag}$ and the extinction curves of Nandy et al. (1976). The optical emission-line strengths are in qualitative agreement with previous measurements (Boyarchuk and Mustel 1964).

\section{ORIGIN OF THE CONTINUUM}

Spectra of novae at minimum light are usually interpreted in terms of emission from an accretion disk surrounding a white dwarf (see Kraft 1964; Bath et al. 


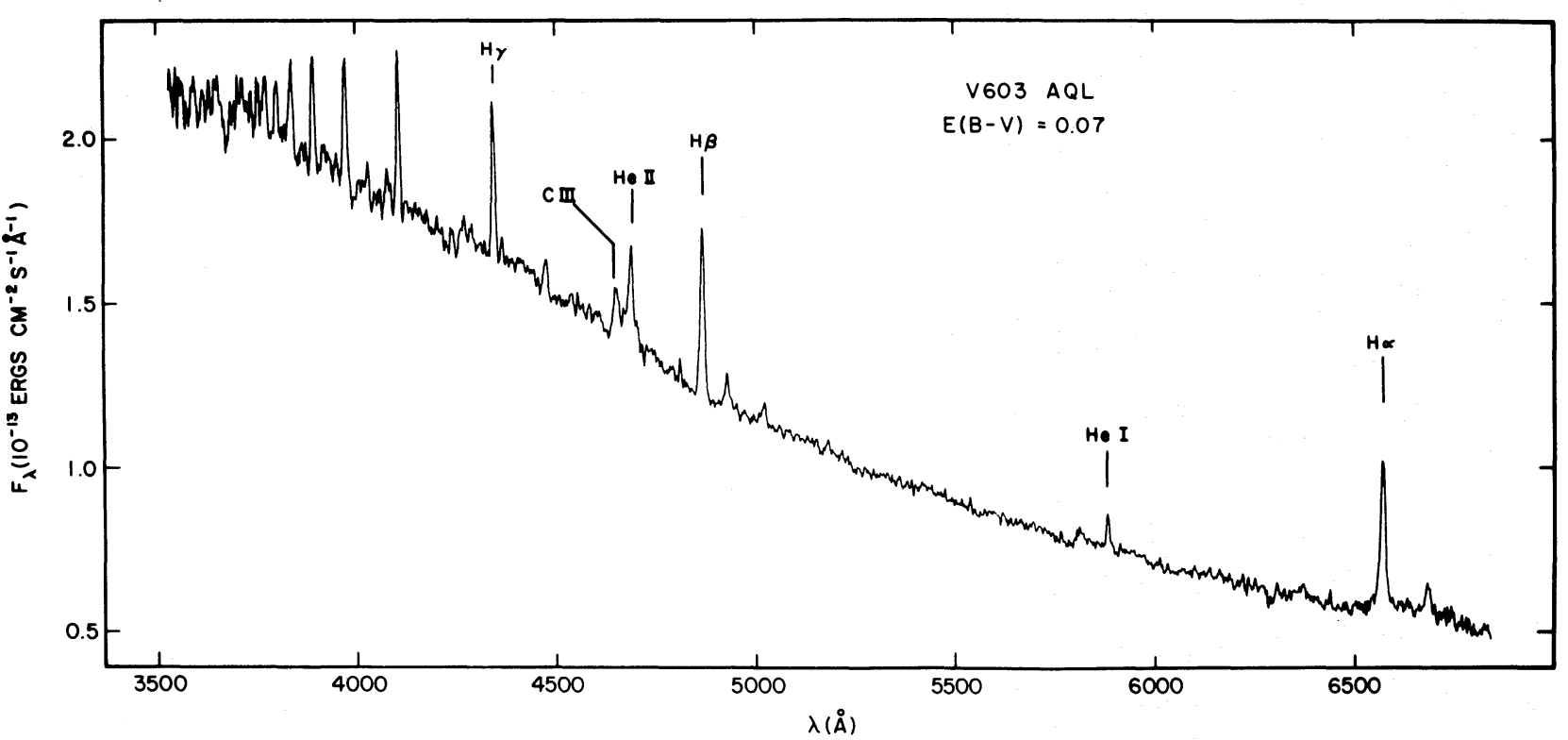

FIG. 2. - The IIDS on the Kitt Peak $2.1 \mathrm{~m}$ telescope was used to obtain optical spectra over the interval $\lambda \lambda 3500-5200 \AA$ at $7 \AA$ resolution, and over the interval $\lambda \lambda 4700-6900 \AA$ at $9 \AA$ resolution. Standard reduction procedures and standard stars were used to calibrate the data. The absolute calibration may contain errors which exceed $10 \%$, although the color calibration should be more accurate than this in the green-red region.

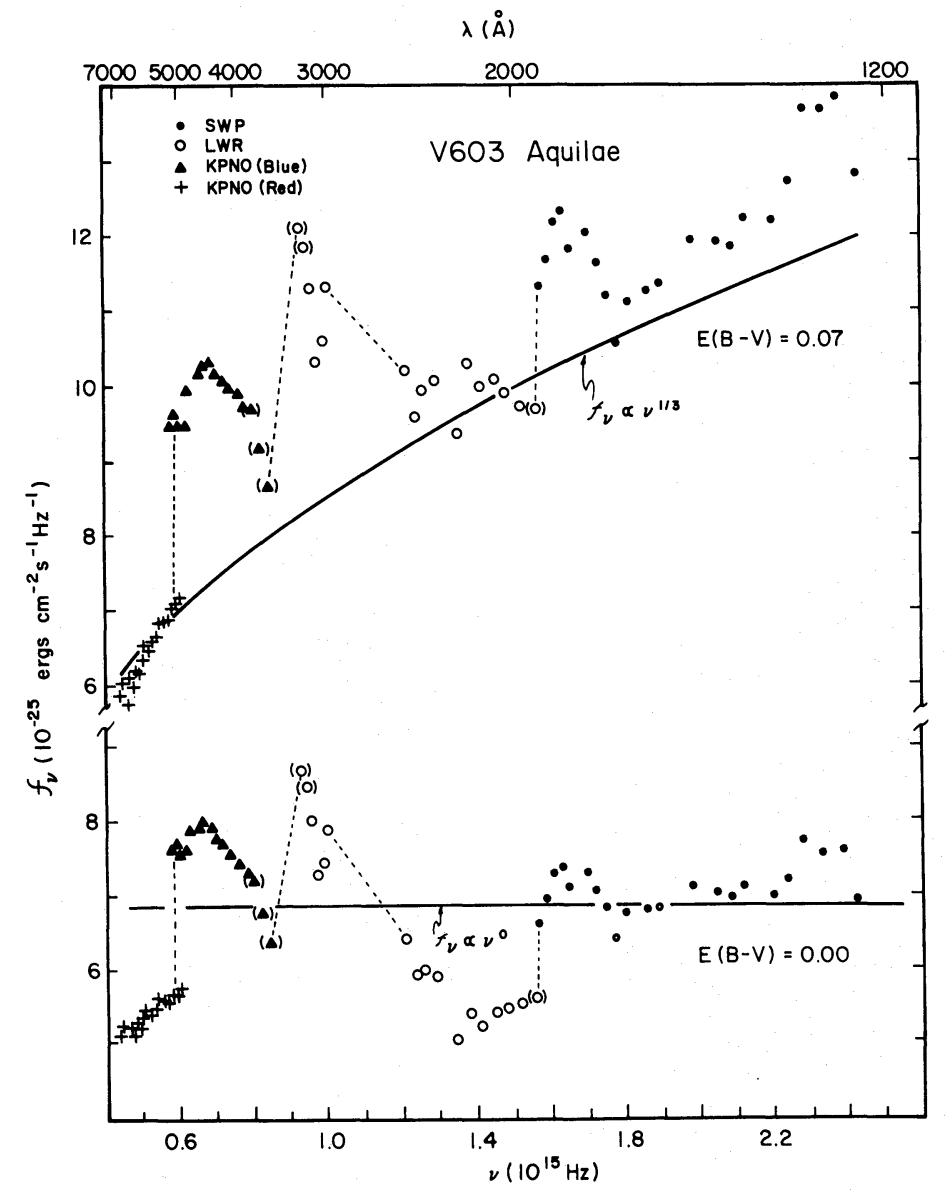

FIG. 3.-The lower panel shows the observed optical-ultraviolet continuous energy distribution. This spectrum is affected by intrinsic variability $(\sim \pm 50 \%)$, uncertainties in absolute calibrations $(\sim \pm 15 \%)$, and interstellar reddening. The upper panel shows the energy distribution after applying an interstellar reddening correction corresponding to $E(B-V)=0.07$ magnitude. This reddening is consistent with the object's galactic position and brings the continuum to the form predicted for optically thick accretion disks, $f_{\nu} \propto \nu^{1 / 3}$. 
TABLE 2

UlTRAVIOLET EMISSION LINES

\begin{tabular}{|c|c|c|c|c|}
\hline Ion & $\lambda(\AA)$ & SWP 5678 & SWP 8756 & Mean \\
\hline $\begin{array}{l}\mathrm{C} \operatorname{IV}^{\mathrm{a}} \ldots \\
\mathrm{C} \mathrm{IV}^{\mathrm{b}} \ldots \\
\end{array}$ & $\begin{array}{l}1549 \\
1549 \\
\end{array}$ & $\begin{array}{l}1.27 \times 10^{-11} \\
15.1 \AA\end{array}$ & $\begin{array}{l}0.83 \times 10^{-11} \\
9.3 \AA\end{array}$ & $\begin{array}{l}1.07 \times 10^{-11} \\
12.2 \AA \\
\end{array}$ \\
\hline 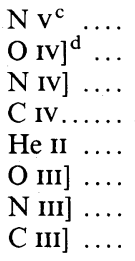 & $\begin{array}{l}1240 \\
1402 \\
1487 \\
1549 \\
1640 \\
1666 \\
1750 \\
1909\end{array}$ & $\begin{array}{r}<0.10 \\
0.32 \\
0.10 \\
1.00 \\
0.14 \\
<0.05 \\
<0.06 \\
<0.03\end{array}$ & $\begin{array}{r}<0.10 \\
0.30 \\
0.20 \\
1.00 \\
0.15 \\
\lesssim 0.09 \\
<0.06 \\
<0.05\end{array}$ & $\begin{aligned}< & 0.10 \\
& 0.31 \\
& 0.15: \\
& 1.00 \\
& 0.14 \\
\lesssim & 0.07: \\
< & 0.06 \\
< & 0.04\end{aligned}$ \\
\hline
\end{tabular}

${ }^{a}$ Flux; ergs s${ }^{-1} \mathrm{~cm}^{-2}$.

${ }^{\mathrm{b}}$ Equivalent width.

${ }^{c}$ Intensity relative to C IV $\lambda 1549$.

${ }^{\mathrm{d}}$ Blending with $\mathrm{Si}$ IV $\lambda 1398$ possible.

TABLE 3

EMISSION LINE INTENSITIES

\begin{tabular}{|c|c|c|c|}
\hline Ion & $\lambda(\AA)$ & $\begin{array}{c}I_{\mathrm{obs}} \times 10^{13} \\
\left(\mathrm{ergs} \mathrm{s} \mathrm{cm}^{-1}\right)\end{array}$ & $\begin{array}{c}I_{\text {cor }} \times 10^{13} \\
\left(\mathrm{ergs} \mathrm{s}^{-1} \mathrm{~cm}^{-2}\right)^{\mathrm{a}}\end{array}$ \\
\hline He I .... & 6678 & 0.87 & 1.15 \\
\hline $\mathrm{H}_{\mathrm{I}} \ldots \ldots$ & 6563 & 4.70 & 6.16 \\
\hline He I $\ldots$ & 5876 & 0.70 & 1.03 \\
\hline $\mathrm{He} \mathbf{I} \ldots$ & 5016 & 0.45 & 0.66 \\
\hline He I .... & 4922 & 0.80 & 1.18 \\
\hline $\mathrm{H} \mathrm{I} \ldots \ldots$ & 4861 & 3.92 & 5.77 \\
\hline H I...... & 4861 & 4.23 & 6.23 \\
\hline He II ... & 4686 & 3.12 & 4.59 \\
\hline $\mathrm{C}$ III $\ldots$ & 4650 & 1.59 & 2.30 \\
\hline He I .... & 4471 & 0.82 & 1.23 \\
\hline $\mathrm{H}$ I...... & 4340 & 3.61 & 5.36 \\
\hline H I...... & 4101 & 3.81 & 5.77 \\
\hline C III .... & 4070 & 0.7: & 1.0: \\
\hline $\mathrm{H} \mathbf{1} \ldots \ldots$ & 3970 & 2.65 & 4.01 \\
\hline H I...... & 3889 & 1.70 & 2.57 \\
\hline He II ... & 1640 & 15 & 27 \\
\hline $\mathrm{C}$ IV .... & 1549 & 107 & 190 \\
\hline $\mathrm{N}$ Iv] $\ldots$ & 1483 & 20: & 30: \\
\hline O Iv] ... & 1402 & 33 & 60 \\
\hline
\end{tabular}

${ }^{\mathrm{a}}$ Corrected for $E(B-V)=0.07$.

1974; Robinson 1976; Warner 1976; Tylenda 1977; Williams 1980). Matter is believed to pass from the faint member of the system onto this disk. Angular momentum is transferred out through the disk, and matter slowly streams onto the surface of the white dwarf. If the disk is optically thick, the matter radiates efficiently and equilibriates at $T_{e} \lesssim 10^{5} \mathrm{~K}$. The observed energy distribution is in good agreement with predictions for such a disk $\left(f_{\nu} \propto \nu^{1 / 3}\right.$; Lynden-Bell 1969) after a correction for a reddening of $E_{B-V}=0.07$ is made (see Fig. 3). Bath, Pringle, and Whelan (1980) have recently found that steady state optically thick models successfully match UV-optical spectra of several dwarf novae.
V603 Aql is well suited for study because its distance and orbital parameters are fairly well known. Warner (1976) estimated masses $M_{1} \approx 0.9 M_{\odot}, M_{2} \approx 0.4 M_{\odot}$, and the inclination $i \approx 15^{\circ}$ from the orbital period and Roche lobe filling arguments. A small inclination is consistent with the optical light curve (Slovak 1981).

A lower limit to the total luminosity radiated by the disk is obtained by integrating the observed energy distribution. Although the true shape of the continuum is uncertain because of errors introduced by interstellar reddening and flickering, the observations suggest

$$
f_{\nu} \approx 1.0 \times 10^{-23}\left(\frac{\nu}{2 \times 10^{15} \mathrm{~Hz}}\right)^{1 / 3} \operatorname{ergs~s}^{-1} \mathrm{~cm}^{-2} \mathrm{~Hz}^{-1} \text {. }
$$

If this continuum turns over at a wavelength $\lambda_{\min }$, the total observed power is

$$
\begin{aligned}
P_{\text {tot }} & =\int_{0}^{\nu_{\text {high }}} f_{\nu} d \nu \\
& =2.6 \times 10^{-9}\left(\frac{\lambda_{\text {min }}}{10^{3} \AA}\right)^{-4 / 3} \operatorname{ergs~s}^{-1} \mathrm{~cm}^{-2} .
\end{aligned}
$$

For both blackbody and bremsstrahlung emitters, the high frequency cutoff is set by the exponential Wien tail appropriate to the inner part of the disk, and $\lambda_{\min }$ is roughly $\left(10^{7.5} \AA \mathrm{K}\right) / T_{\max }$. Since the energy distribution has not turned over at $\lambda=10^{3} \AA, T_{\max } \gtrsim 10^{4.5} \mathrm{~K}$. Strong C IV, Si IV, and He II lines and the absence of N V 1240 suggest that $T_{\max }$ is not much larger than $\sim 10^{5.2} \mathrm{~K}$, or $\lambda_{\text {min }} \sim 200 \AA$. At a distance of 380 pc the total luminosity is

$$
L_{\text {tot }}=3.6 \times 10^{35}\left(\frac{\lambda_{\min }}{200 \AA}\right)^{-4 / 3} \operatorname{ergs~s}^{-1} .
$$


If this luminosity is generated in the accretion disk, a standard expression (see Bath, Pringle, and Whelan 1980) shows that the mass transfer rate is

$$
\dot{M} \sim 10^{18.2}\left(\frac{\lambda_{\min }}{200 \AA}\right)^{-4 / 3}\left(\frac{R}{10^{8.7} \mathrm{~cm}}\right)^{-1} \mathrm{~g} \mathrm{~s}^{-1} .
$$

Stability arguments suggest that the disk can extend to an outer radius equal to $\sim$ one-third the binary separation. This outer radius is $R_{\text {out }} \sim 10^{10.6} \mathrm{~cm}$ for the V603 Aql system. Detailed calculations (Williams 1980) show that, for the mass transfer rate deduced above, the disk will be optically thick to continuous absorption throughout.

\section{THE LINE SPECTRUM}

\section{a) General Overview}

The fact that lines are seen in emission against the continuum originating in an optically thick disk demands that they be formed in a region which is either larger or hotter than the disk. This emission-line region could be a large optically thin annulus outside the disk (Williams 1980) or a hot corona suspended above the disk (Warner 1976). We argue that the lines are formed in a corona with radius comparable to the binary separation, characterized by a density $N \sim 10^{9}-10^{11} \mathrm{~cm}^{-3}$ and mass $\sim 10^{22} \mathrm{gm}$.

\section{b) The Emission-Line Region}

The line spectrum of V603 Aql is dominated by permitted lines of $\mathrm{H}, \mathrm{He}, \mathrm{C}$, and $\mathrm{Si}$ (see Table 3). Neither optical forbidden lines (e.g., [O III] $\lambda \lambda 5007$, 4959) nor the ultraviolet intercombination line $O$ III] $\lambda 1666$ are detected, even though the presence of strong He I lines show that $\mathrm{O}^{++}$should be present. The absence of these lines can be understood as a consequence of high electron densities; if $N_{e} \gtrsim 10^{9.0} \mathrm{~cm}^{-3}$, excited metastable states reach their LTE population and the volume line emissivity no longer increases as $N^{2}$ (see Osterbrock 1974). Some intercombination lines are seen; these include the blend at $\lambda 1400 \AA$, which may contain O IV] $\lambda 1402 \AA$, and the feature at $\lambda 1485 \AA$, which we identify as $\mathrm{N}$ IV] $\lambda 1486$. As the nitrogen line has a critical density of $N_{\text {crit }}=10^{10.5} \mathrm{~cm}^{-3}$, the bulk of the coronal material must be characterized by a density $10^{9} \mathrm{~cm}^{-3} \leq N \leq 10^{11} \mathrm{~cm}^{-3}$.

The emission measure of a recombination line such as $\mathrm{H} \beta$ relates the $\mathrm{H} \beta$ flux to the density and volume (see Osterbrock 1974). If the Balmer lines are formed by recombination in a region of density $N_{e}$, temperature $T_{e}$, and volume $V$, the total power radiated in a line such as $\mathrm{H} \beta$ will be

$$
P(\mathrm{H} \beta)=N\left(\mathrm{H}^{+}\right) 4 \pi j\left(\mathrm{H} \beta, N_{e}, T_{e}\right) V N_{e}
$$

where $j\left(H \beta, N_{e}, T_{e}\right)$ is the emission coefficient (ergs $\mathrm{s}^{-1}$ $\left.\mathrm{cm}^{3} \mathrm{sr}^{-1}\right)$. Detailed radiative transfer calculations, which include the effects of large optical depths and high densities (Netzer 1975, 1977; Krolik and McKee 1978; Drake and Ulrich 1980) show that the $\mathrm{H} \beta$ emission coefficient can be expressed as

$$
\begin{aligned}
4 \pi j\left(\mathrm{H} \beta, N_{e}, T_{e}\right) \sim 1.2 \times 10^{-25} T_{4}^{-0.7} f\left(N_{e}, \tau\right) & \\
& \quad \operatorname{ergs~s}^{-1} \mathrm{~cm}^{-3},
\end{aligned}
$$

where $T_{e}=T_{4} \times 10^{4} \mathrm{~K}$ and $f\left(N_{e}, \tau\right) \sim 10^{0 \pm 1}$ is a modeldependent factor. If the emission-line region has a characteristic density $N \sim 10^{10} \mathrm{~cm}^{-3}$, then the observed power in $\mathrm{H} \beta$ corresponds to a volume

$$
V=8.2 \times 10^{35} \mathrm{~cm}^{3} T_{4}^{0.7} f\left(N_{e}, \tau\right)^{-1} N_{10}^{-2},
$$

where $N_{10}=N /\left(10^{10} \mathrm{~cm}^{-3}\right)$. If this mass is uniformly distributed over a sphere, then the corresponding radius is $r \sim 10^{11.8} N_{10}^{-2 / 3} \mathrm{~cm}$, comparable to the binary separation (Warner 1976). The total mass of this region is

$$
\begin{aligned}
M_{\mathrm{em}}= & 10^{22.3}\left(\frac{D}{380 \mathrm{pc}}\right) \\
& \times T_{4}^{0.7}\left(\frac{V}{10^{35.9} \mathrm{~cm}^{3}}\right)^{1 / 2} f\left(N_{e}, \tau\right)^{-1 / 2} \mathrm{~g} .
\end{aligned}
$$

At the mass transfer rate deduced above, $\dot{M} \sim 10^{18}$ $\mathrm{gm} \mathrm{s}^{-1}$, the emission line region can be replenished in a time $\tau_{\mathrm{acc}} \sim M_{\mathrm{em}} / \dot{M} \sim 10^{4.3} \mathrm{~s}$. For comparison, the radiative cooling time of the gas is

$$
\tau_{\text {cool }} \simeq 3 / 2 N k T_{e} /\left[\Lambda\left(T_{e}\right) N^{2}\right] \sim 10^{0.7} N_{10}^{-1} \mathrm{~s},
$$

where $\Lambda\left(T_{e}\right)=4 \times 10^{-23} \mathrm{ergs} \mathrm{s}^{-1} \mathrm{~cm}^{+3}$ is the radiative cooling function for $T_{e}=10^{4} \mathrm{~K}$ (Raymond, Cox, and Smith 1976; see also Warner 1976). Since $\tau_{\text {cool }}$ is much shorter than the replenishment time, the emission-line region must be constantly heated, either by dissipation of shocks as in the solar corona or by the radiation field of the disk or white dwarf.

The density derived here is much smaller than the canonical value of $N \sim 10^{13} \mathrm{~cm}^{-3}$ (see Warner 1976). This higher value was obtained from the emission measure by identifying the emitting volume as a region similar in size to the accretion disk. Such a high density (and the correspondingly small emitting volume) are inconsistent with the presence of the $\mathrm{N}$ IV] and $\mathrm{O}$ IV] intercombination lines, however.

In principle, the position of the emission region may be deduced from the emission-line widths because the velocity field of an accretion disk or gas stream should be highly ordered. Matter at a radius $r$ in the accretion 
disk will move in an approximately circular orbit with velocity

$$
V \approx 3.6 \times 10^{3}\left(\frac{M}{M_{\odot}}\right)^{1 / 2}\left(\frac{r}{10^{9} \mathrm{~cm}}\right)^{-1 / 2} \mathrm{~km} \mathrm{~s}^{-1}
$$

while gas in free fall will have a velocity $(2)^{1 / 2} \approx 1.4$ times larger. The projected velocity will be a factor of $\sin i=0.26$ smaller if $i=15^{\circ}$. This expression allows us to unambiguously relate the line width to the smallest radius of line formation.

Unfortunately, the line widths in the present ultraviolet and optical spectra are largely set by the instrumental resolution. The half-width at zero-intensity $\left(V_{\text {HWZI }}\right)$ is $\lesssim 630 \mathrm{~km} \mathrm{~s}^{-1}$ for the H I and He I optical lines. Application of the projection factor gives an intrinsic velocity $V \lesssim 2500 \mathrm{~km} \mathrm{~s}^{-1}$, corresponding to an orbit radius $r>2 \times 10^{9} \mathrm{~cm}$. Unpublished 107" Reticon spectra show $\mathrm{H} \alpha$ to have a HWHM of $175 \mathrm{~km} \mathrm{~s}^{-1}$ and a HWZI of $450 \mathrm{~km} \mathrm{~s}^{-1}$. The corrected velocities of $\sim 670$ $\mathrm{km} \mathrm{s}^{-1}$ and $1730 \mathrm{~km} \mathrm{~s}^{-1}$, respectively, correspond to $r \approx 3 \times 10^{10} \mathrm{~cm}$ for the typical line forming region and $r \approx 4 \times 10^{9} \mathrm{~cm}$ for the innermost portion of the corona. These limits, together with the estimate of the emitting volume presented above, identifies the emission-line region as a dilute gas with dimensions comparable to the binary separation.

\section{c) Emission-Line Intensities}

In general, emission-line spectra can be easily treated in two limiting cases: the LTE and the nebular approximations. The former assumes that the level populations are held to their values in thermodynamic equilibrium and requires that collisions proceed much more rapidly than radiative processes, or $N_{e}>A_{21} / q_{21}$ $\sim 10^{16} \mathrm{~cm}^{-3}$ for $A_{21} \sim 10^{9} \mathrm{~s}^{-1}$ and a collisional rate $q_{21} \sim 10^{-7} \mathrm{~cm}^{3} \mathrm{~s}^{-1}$, typical values for UV resonance lines. The presence of intercombination lines shows that the gas in the corona of V603 Aql cannot be dense enough for LTE to apply.

The nebular approximation applies when the density is low enough for collisional de-excitation of even strongly trapped resonance lines to be unimportant; all photons escape. A resonance line formed at a depth $\tau_{0}$ will scatter $\langle N\rangle \sim \tau_{0}$ times before escaping (Auer 1968; Avery and House 1968). The requirement that the photon escape before being collisionally de-excited sets an upper limit to the density of $N_{e} \lesssim A_{21} /\left(\langle N\rangle q_{21}\right) \sim$ $10^{16} / \tau_{0} \mathrm{~cm}^{-3}$. Model calculations show that Ly $\alpha$ and C IV 1549 can have optical depths $\tau_{0} \sim 10^{4}-10^{6}$ for thermal broadening, so densities $N \lesssim 10^{10} \mathrm{~cm}^{-3}$ are needed for the nebular approximation to apply. Although the coronal gas is likely to have a density near this upper limit, the nebular approximation should still be valid since line optical depths will be reduced by turbulence and macroscopic motion of the gas, and because of the strongly self-correcting thermostat effect introduced by the fact that the strong UV lines are important coolants for the gas (see Shuder and MacAlpine 1979).

A detailed calculation of line intensities should also include the resonance fluorescent effects caused by the disk continuum. These effects could be important if the dilution factor in the emitting region is large.

\section{i) Balmer Lines}

Table 4 lists the relative intensities of the six strongest Balmer lines. The table also gives theoretical relative intensities for nebular conditions (case $\mathrm{B}, T_{e}=10^{4} \mathrm{~K}$, $N=10^{5} \mathrm{~cm}^{-3}$; Brocklehurst 1971). The Balmer decrement is much flatter than predicted by these calculations, as is commonly the case in spectra of cataclysmic variables (Stockman et al. 1977; Kiplinger 1978, 1979; Williams 1980). As both Stockman et al. and Williams point out, this Balmer decrement is consistent with LTE emission at $6000 \mathrm{~K}$, but it is difficult to see how LTE could be maintained for the low densities estimated above. Continuum fluorescence (case C) will affect Balmer lines if Lyman lines absorb a continuum width of $v \gtrsim 10^{2} \mathrm{~km} \mathrm{~s}^{-1}$. Case C Balmer decrements are steeper, not shallower, than case B, however (Aller 1956).

Drake and Ulrich (1980) have studied hydrogenic emission spectra for a hot dense gas similar to the corona of V603 Aql. Their models are parameterized by the density, Ly $\alpha$ optical depth, electron temperature, and a photon ionization parameter $R_{\text {IC }}$ defined as

$$
\begin{aligned}
R_{\mathrm{IC}} & =4 \pi \int_{\nu_{1}}^{\infty} a_{\nu} F_{\nu} / h \nu d \nu \\
& =\frac{N_{\mathrm{H}^{+}}}{N_{\mathrm{H}^{0}}} N_{e} \alpha \approx 10^{3.5},
\end{aligned}
$$

where $\nu_{1}$ is the $\mathrm{H}^{0}$ photoionization threshold, the photoionization cross section is $a_{\nu}=6.3 \times 10^{-18}\left(\nu / \nu_{1}\right)^{-3}$,

TABLE 4

HydROGEN LINE SPECTRUM

\begin{tabular}{ccccc}
\hline \hline $\begin{array}{c}\lambda(\AA) \\
(1)\end{array}$ & $\begin{array}{c}\text { Transition } \\
(2)\end{array}$ & $\begin{array}{c}I_{\text {obs }} \\
(3)\end{array}$ & $\begin{array}{c}I_{\mathrm{B}}^{\mathrm{a}} \\
(4)\end{array}$ & $\begin{array}{c}I_{\mathrm{DU}}^{\mathrm{b}} \\
(5)\end{array}$ \\
\hline $6563 \ldots$ & $3-2$ & 0.99 & 2.80 & 0.95 \\
$4861 \ldots$ & $4-2$ & 1.00 & 1.00 & 1.00 \\
$4340 \ldots$ & $5-2$ & 0.86 & 0.47 & 0.82 \\
$4101 \ldots$ & $6-2$ & 0.92 & 0.29 & 0.56 \\
$3970^{\mathrm{c}} \ldots$ & $7-2$ & 0.64 & 0.16 & 0.42 \\
$3889^{\mathrm{d}} \ldots$ & $8-2$ & 0.41 & 0.11 & 0.28 \\
\hline
\end{tabular}

\footnotetext{
a Nebular predictions from Brocklehurst 1971.

b Predictions for $N_{e}=10^{13.2} \mathrm{~cm}^{-3}$, from Drake and Ulrich 1980.

${ }^{\mathrm{c}}$ Possible contribution from $\mathrm{He}$ i $\lambda 3964,4{ }^{1} P-2{ }^{1} S$.

${ }^{\mathrm{d}}$ Possible contribution from He I $\lambda 3888,3{ }^{3} \mathrm{P}-2{ }^{3} \mathrm{~S}$.
} 
and the recombination coefficient is $\alpha\left(T_{e}, N_{e}\right) \approx 2.6 \times$ $10^{-13} T_{4}^{-0.8}$. Column 5 of Table 4 summarizes one of the better fitting Drake and Ulrich models; with $R_{\mathrm{IC}}=0.03$, $\tau_{\text {Ly } \alpha}=10^{3}, T_{e}=10^{4} \mathrm{~K}, N_{e}=10^{13.2} \mathrm{~cm}^{-3}$. The fit to the first three Balmer lines is quite good but grows worse with higher $n$ lines. However, the first is encouraging because the grid of models is fairly coarse and optimization is difficult. The model dependent factor for the $\mathrm{H} \beta$ emissivity is $f\left(N_{e}, T_{e}, \tau\right)=0.26$ for these parameters. At least part of this agreement must be fortuitous because the value of the photon ionization parameter indicated by the hydrogen neutral fraction (see the calculations in $\S \mathrm{V}$ ) is several orders of magnitude larger than any of the Drake and Ulrich models, and the density of the corona is likely to be much smaller.

The large photon density will affect emission-line ratios in the coronal gas because of the importance of stimulated emission. The ratio of stimulated to spontaneous emission rates for $\mathrm{Pa} \alpha$, a transition which strongly affects the $\mathrm{H} \alpha / \mathrm{H} \beta$ ratio, will be $B_{43} / A_{43}=\left(c^{2} / 2 h \nu^{3}\right) I$ $\approx 7.4 \times 10^{21} r^{-2}$, where $r$ is the distance from the accretion disk whose continuum is assumed to be given by equation (1). The stimulated and spontaneous emission rates are comparable as distant as $\sim 10^{11} \mathrm{~cm}$ from the disk. Detailed calculations of the effects of the disk radiation field upon the Balmer lines are beyond the scope of this paper, but stimulated emission should tend to bring the level populations to LTE at lower than expected densities.

\section{ii) Helium Lines}

Table 5 summarizes the $\mathrm{He}$ I line intensities. The predictions for a recombination spectrum from an optically thin low-density gas are also listed (Brocklehurst 1972). The discrepancy between the observed and predicted spectra resembles the Balmer line case; i.e., the observed relative intensities show the smaller range. The Balmer lines may be explained by trapping of resonance line radiation. This explanation cannot apply to the $\mathrm{He}$ I lines because He I $\lambda 584$ (and other resonance lines) is destroyed by photoionization of hydrogen (Ferland 1980).

Although detailed calculations of the $\mathrm{He}$ I spectrum of V603 Aql are beyond the scope of this paper, it may be noted that three additional processes-dielectronic and three-body recombination and collisional excitation from the metastable $2{ }^{3} s$ state will compete with radiative recombination in formation of the $\mathrm{He} \mathrm{I}$ lines. These processes are unimportant in low-density gaseous nebulae, and their effects are not reflected in Brocklehurst's calculations. Quantitative estimates based on published rate constants (Burgess and Summers 1976; Feldman and MacAlpine 1978) suggest that the three additional processes acting in consort exceed the radiative recombination rate by a factor of about 2 . It is not known
TABLE 5

He I LINE SPECTRUM

\begin{tabular}{llll}
\hline \hline$\lambda(\AA)$ & Transition & $I_{\text {obs }}$ & $I_{\text {pred }}$ \\
\hline $6678 \ldots$ & $2{ }^{1} P^{0}-3{ }^{1} D$ & 0.93 & 0.78 \\
$5876 \ldots$ & $2{ }^{3} P^{0}-3^{3} D$ & 0.83 & 2.58 \\
$5016 \ldots$ & $2{ }^{1} S-3{ }^{1} P^{0}$ & 0.53 & 0.59 \\
$4922 \ldots$ & $2{ }^{1} P^{0}-4{ }^{1} D$ & 0.96 & 0.27 \\
$4471 \ldots$ & $2{ }^{3} P^{0}-4^{3} D$ & 1.00 & 1.00 \\
\hline
\end{tabular}

TABLE 6

He II LINE SPECTRUM

\begin{tabular}{ccrc}
\hline \hline$\lambda(\AA)$ & Transition & $I_{\text {obs }}$ & $I_{\text {pred }}$ \\
\hline $1640 \ldots$ & $3-2$ & 5.9 & 6.80 \\
$4686 \ldots$ & $3-4$ & 1.0 & 1.00 \\
$5412 \ldots$ & $7-4$ & $\lesssim 0.1$ & 0.08 \\
\hline
\end{tabular}

whether these processes possess the specificity to, for example, increase $4922 \AA$ line relative to the $4471 \AA$ line.

Table 6 lists intensities of the three strongest He II lines. Theoretical intensities are taken from Seaton $(1978 a)$. The $\lambda 1640 / \lambda 4686$ intensity ratio is quite insensitive to density and only weakly sensitive to temperature. It is no surprise that the $\mathrm{He}$ II spectrum is in substantial agreement with predictions because trapped He II Ly $\alpha$ is ineffective in populating excited states thanks to its rapid destruction by either photoionization of $\mathrm{H}^{0}$ or $\mathrm{He}^{0}$ or by conversion to Bowen $\mathrm{O}$ III lines (Weymann and Williams 1969). Dielectronic recombination will not affect this one-electron ion, of course. Three-body recombination will also be negligible if $N \sim$ $10^{10} \mathrm{~cm}^{-3}-10^{11} \mathrm{~cm}^{-3}$ (Burgess and Summers 1976).

\section{iii) $\mathrm{C}, \mathrm{N}$, and $\mathrm{O}$ Lines}

The feature at $\lambda=4648 \pm 3 \AA$ is assigned to $C_{\text {III }}$ RMT $13 p{ }^{3} P-3 s{ }^{3} S$, a multiplet with components at $\lambda \lambda 4647,4650$, and $4651 \AA$. The $\mathrm{N}$ III multiplet $3 p$ ${ }^{2} P^{0}-3 d^{2} D \lambda \lambda 4634,4631,4642 \AA$, often identified as a contributor in lower resolution scans, is not present.

The carbon recombination spectrum has been studied by Pengelly (1963, as quoted in Seaton 1978b) and by Storey (1981). The $\lambda 4650$ blend is predicted to be the strongest $\mathrm{C}$ III line, with progressively weaker lines at $\lambda \lambda 4070 \AA$ and $4187 \AA$, when radiative recombination dominates. A feature may be present at the expected position of the $\lambda 4070$ line, and its intensity relative to $\lambda 4650$ is in fair agreement with the value predicted for some conditions. Storey (1981) has shown that dielectronic recombination through low lying autoionizing states is $\sim 4$ times faster than radiative recombination for nebular temperatures and the low-density limit $\left(T_{e} \sim\right.$ $10^{4} \mathrm{~K}$; Storey 1981; Aldrovandi and Pequignot 1973). Effective dielectronic recombination coefficients for the 
optical C III lines have not been published but can be roughly estimated by assuming that dielectronic recombination increases both the effective and total recombination by the same factor. Scaling Pengelly's case B results we find

$$
\alpha_{\text {eff }}(\lambda 4650) \sim 9 \times 10^{-12} T_{4}^{-0.73} \mathrm{~cm}^{3} \mathrm{~s}^{-1} .
$$

This rate is uncertain to a factor of $\sim 3$ because of both uncertainties intrinsic to Storey's analysis and our extrapolation to densities much higher and radiation fields much stronger than the regimes considered by Storey.

The C III $\lambda 4650 / C$ IV $\lambda 1549$ intensity ratio can be used to estimate the electron temperature accurately despite these uncertainties. Assuming that $\lambda 1549$ is collisionally excited while $\lambda 4650$ is formed as described above, and that both lines are effectively optically thin, their intensity ratio is related to the temperature by

$$
\frac{I(\lambda 1549)}{I(\lambda 4650)}=1.3 \times 10^{5} T_{4}^{0.23} e^{-9.296 / T_{4}} .
$$

The reddening corrected intensity ratio of $I(1549) /$ $I(4650)=83$ corresponds to $T_{e} \approx 1.3 \pm 0.3 \times 10^{4} \mathrm{~K}$, where the uncertainty corresponds to the factor of $\sim 5$ error introduced by uncertainties in the intensities and recombination coefficient.

This low temperature has two implications. First, it confirms our suspicion that the emission-line region has dimensions much larger than the accretion disk. Were the emitting material suspended only above the disk then the $\mathrm{C}$ IV line would appear in absorption since the disk radiates as a hotter blackbody. Second, it seems much more likely that the emission-line region is photoionized rather than the result of mechanical heating. The deduced temperature is typical of that found in $\mathrm{C}^{+3}$ zones of photoionized nebulae but much smaller than that produced in a coronal equilibrium situation $\left(T_{\mathrm{C}^{+3}} \geq\right.$ $10^{5} \mathrm{~K}$; Nussbaumer and Storey 1975).

The abundances of $\mathrm{He}, \mathrm{C}, \mathrm{N}$, and $\mathrm{O}$ can be roughly estimated from this temperature and the ultraviolet lines. If $\mathrm{C}$ IV $\lambda 1549$ is collisionally excited and $\mathrm{He}$ II $\lambda 1640$ formed by radiative recombination, then their intensity ratio is related to the $\mathrm{C}^{+3} / \mathrm{He}^{+2}$ ionic abundance ratio by

$$
\frac{N\left(\mathrm{C}^{+3}\right)}{N\left(\mathrm{He}^{+2}\right)}=1.97 \times 10^{-6} T_{4}^{-0.33} e^{9.296 / T_{4}} \frac{I(\lambda 1549)}{I(\lambda 1640)},
$$

where we have used the rates given by Seaton $(1978 a)$ and Harrington et al. (1980).

To compare this ionic ratio with total elemental abundances, unseen stages of ionization must be considered. The ionization potentials suggest that there can be little
$\mathrm{C}^{+4}$ in the $\mathrm{He}^{+2}$ zone since the small $\mathrm{N} v \lambda 1240 / \mathrm{N}$ Iv] $\lambda 1486$ intensity ratio suggests that $\mathrm{N}^{+4}$ is absent. Some $\mathrm{C}^{+3}$ can exist in the $\mathrm{He}^{+}$zone, however, and so

$$
\frac{N(\mathrm{C})}{N(\mathrm{He})} \lesssim \frac{N\left(\mathrm{C}^{+3}\right)}{N\left(\mathrm{He}^{+2}\right)}=\operatorname{dex}\left(-1.8_{-0.5}^{+1.0}\right),
$$

consistent with the solar $\mathrm{C} / \mathrm{He}$ ratio of $10^{-2.2}$ (Lambert 1978).

The $\mathrm{C}^{+3} / \mathrm{N}^{+3}$ ionic abundance ratio is related to the $\mathrm{C}$ IV, $\mathrm{N}$ IV] intensities by

$$
\frac{N\left(\mathrm{C}^{+3}\right)}{N\left(\mathrm{~N}^{+3}\right)}=0.19 e^{0.39 / T_{4}} \frac{I(\lambda 1549)}{I(\lambda 1486)} \approx 10^{0.2 \pm 0.3},
$$

where the uncertainty is largely due to errors in the intensity of the $\mathrm{N}$ IV] line. This ionic ratio should be close to the abundance ratio since the ionization potentials agree quite well. Nitrogen may be enhanced relative to carbon by $\gtrsim 0.4$ dex over the solar ratio of $\mathrm{C} / \mathrm{N}=$ $10^{0.67}$ (Lambert 1978). The enhancement could be even larger if the coronal density is close to the critical density of the intercombination line $\left(N_{\text {crit }}=10^{10.5} \mathrm{~cm}^{-3}\right)$.

The feature at $\lambda 1400 \AA$ must be largely O Iv] $\lambda 1402$. The absence of significant C III] $\lambda 1909$ emission demands that there is little $\mathrm{C}^{+2}$ in the corona (this absence cannot be due to high densities because the $\mathrm{N} \mathrm{IV]} \mathrm{line} \mathrm{is}$ quite strong), and consideration of the relevant ionization potentials suggests that $\mathrm{Si}$ IV $\lambda 1397$ must also be absent. The $\mathrm{O}^{+3} / \mathrm{C}^{+3}$ ratio is related to the $\left.\mathrm{O} \mathrm{IV}\right] / \mathrm{C}$ IV intensities by

$$
\frac{N\left(\mathrm{O}^{+3}\right)}{N\left(\mathrm{C}^{+3}\right)}=6.0 e^{0.95 / T_{4}} \frac{I(\lambda 1402)}{I(\lambda 1549)}=10^{0.5 \pm 0.3} .
$$

This is consistent with the solar abundance ratio of $10^{0.25}$ (Lambert 1978), since a large uncertainty is introduced by the fact that the ionization potential match is less than ideal. In this calculation we have employed the new $O$ IV] $\lambda 1402$ collision strength recently computed by Henry (1982). The value of $\Omega(\lambda 1402)=4.04$ is in excellent agreement with that inferred by Wills and Netzer (1979) in their analysis of quasar spectra.

\section{A MODEL CORONA}

\section{a) Model Requirements and Constraints}

The ultraviolet and optical observations of V603 Aql have set several constraints on the origin of the emission lines. The presence of intercombination lines suggests densities $10^{9} \mathrm{~cm}^{-3} \leq N \leq 10^{11} \mathrm{~cm}^{-3}$, and the emission measure shows that the region is large $\left(r \sim 10^{11}-10^{12}\right.$ $\mathrm{cm})$. The low temperature of the $\mathrm{C}^{+3}$ zone deduced from C III and C IV emission lines has two implications. 
First, the chemical composition of the gas as indicated by the relative intensities of the emission lines is largely consistent with a solar mixture. The low temperature also strongly suggests that the gas is photoionized. Photoionization is also an attractive source of heating and ionization because the coronal demands on the energy budget of the system are not great; together all optical and ultraviolet lines carry a luminosity of $\sim 10^{33}$ ergs $\mathrm{s}^{-1}$ (see Table 3), while the total power available in radiation is $L \sim 10^{35}$ ergs s$^{-1}$.

Three sources of ionizing radiation must be considered in models of the coronal gas (see also the discussions by Tylenda 1977 and Bath, Pringle, and Whelan 1980). The disk itself radiates a luminosity

$$
\begin{aligned}
L_{\text {disk }}= & 1.3 \times 10^{35}\left(\frac{\dot{M}}{10^{18} \mathrm{~g} \mathrm{~s}^{-1}}\right)\left(\frac{M}{M_{\odot}}\right) \\
& \times\left(\frac{R}{5 \times 10^{8} \mathrm{~cm}}\right)^{-1} \operatorname{ergs~s}^{-1}
\end{aligned}
$$

This continuum has the form

$$
f_{\nu} \sim \nu^{1 / 3} e^{-h \nu / k T_{\max }}
$$

where

$$
\begin{aligned}
T_{\max }= & 10^{5}\left(\frac{\dot{M}}{10^{18} \mathrm{~g} \mathrm{~s}^{-1}}\right)^{1 / 4} \\
& \times\left(\frac{M}{M_{\odot}}\right)^{1 / 4}\left(\frac{R}{5 \times 10^{8} \mathrm{~cm}}\right)^{-3 / 4} \mathrm{~K}
\end{aligned}
$$

Our observations are completely consistent with this view.

A second source of hard radiation has been detected by Becker and Marshall (1981) in their Einstein observations of this old nova. They find a hard continuum with a $0.15-4.5 \mathrm{keV}$ continuum luminosity of $1.3 \times 10^{32}$ ergs $\mathrm{s}^{-1}$. If this is interpreted as an optically thin $10 \mathrm{keV}$ bremsstrahlung spectrum (see Córdova, Mason, and Nelson 1981), then the total luminosity of this source is $L \simeq 3 \times 10^{32}$ ergs s$^{-1}$.

Models of the accretion flow onto the surface of the white dwarf (Pringle 1977; Pringle and Savonije 1979) predict a boundary layer emitting a luminosity equal to $L_{\text {disk }}$ but as a blackbody with temperature

$$
\begin{aligned}
T_{\mathrm{BL}}= & 5 \times 10^{5}\left(\frac{\dot{M}}{10^{18} \mathrm{~g} \mathrm{~s}^{-1}}\right)^{2 / 9}\left(\frac{M}{M_{\odot}}\right)^{1 / 3} \\
& \times\left(\frac{R}{5 \times 10^{8} \mathrm{~cm}}\right)^{-7 / 9} \mathrm{~K} .
\end{aligned}
$$

Apparently this boundary layer emission has been suppressed in V603 Aql, since the bulk of this energy $\left(\sim 10^{35}\right.$ ergs $\left.\mathrm{s}^{-1}\right)$ should lie within the Einstein IPC bandwidth, while Becker and Marshall (1981) detect only $\sim 10^{32}$ ergs s $^{-1}$. Models considered below will treat cases both with and without the predicted boundary layer.

\section{b) Specific Models}

A photoionization model is parameterized by the luminosity and character of the radiation field, the density and chemical composition of the gas, and the dimensions of the system. Once these are set the equations of statistical and thermal equilibrium are solved to predict an emission-line spectrum.

Calculations are performed with the program described by Ferland and Truran (1981). All important $X$-ray ionization effects (see Davidson and Netzer 1979; Halpern and Grindlay 1980) are included, together with processes affecting more neutral material (e.g., charge exchange, secondary ionization by suprathermal electrons). The radiative and dielectronic recombination rates published by Aldrovandi and Pequignot 1973) have been supplemented by the dielectronic recombination rates computed by Storey (1981) and the three-body rates given by Burgess and Summers (1976). All resonance line photons (e.g., C IV $\lambda 1549$ ) are assumed to escape without suffering collisional de-excitation. This approximation is valid if the density is as low as estimated above $\left(N \sim 10^{9}\right.$ to $\left.10^{11} \mathrm{~cm}^{-3}\right)$, and the lines are broadened by Keplerian motions.

The fact that the emission lines carry a luminosity $\left(\sim 10^{33}\right.$ ergs s $\left.^{-1}\right)$ which is small compared to the available continuum luminosity $\left(\sim 10^{35}\right.$ ergs $\left.\mathrm{s}^{-1}\right)$ suggests that the gas either has a small covering factor or is matter bounded (optically thin to ionizing radiation) (or both). The absence of significant C III] $\lambda 1909$ in our spectra and Mg II $\lambda 2798$ emission (Dreschsel et al. 1981) suggests that the gas is optically thin to soft $(\nu \lesssim 2-4$ ryd) radiation, while the fact that both $\mathrm{He}$ II and $\mathrm{He} I$ lines are present suggests that the gas is optically thick to $\nu>4$ ryd photons (see Hummer and Seaton 1964).

In the following calculations the outer radius of the gas is set by stopping the radial integrations when the predicted C IV $\lambda 1549 / \mathrm{He}$ II $\lambda 1640$ ratio reaches its observed value. The inner radius is effectively zero because the gas close to the disk is very highly ionized and produces little optical or ultraviolet emission. A constant density of $N_{\mathrm{H}}=10^{10} \mathrm{~cm}^{-3}$ is assumed. Tests shows that details of the predicted emission-line spectrum do not greatly change for densities within the range $10^{9} \leq$ $N_{\mathrm{H}} \leq 10^{11} \mathrm{~cm}^{-3}$.

Models with two different radiation fields and chemical composition will be considered. As mentioned above, the predicted boundary layer emission is apparently absent in V603 Aql. We consider both models ionized 
TABLE 7

\begin{tabular}{|c|c|c|c|c|c|c|}
\hline $\begin{array}{l}\text { Ion } \\
\text { (1) }\end{array}$ & $\begin{array}{c}\lambda(\AA) \\
(2)\end{array}$ & $\begin{array}{l}I_{\text {obs }} \\
\text { (3) }\end{array}$ & $\begin{array}{c}\text { Giant }^{\mathrm{a}} \\
I_{\mathrm{A}} \\
(4)\end{array}$ & $\begin{array}{c}\text { Solar }^{\mathrm{a}} \\
I_{\mathrm{A}} \\
(5)\end{array}$ & $\begin{array}{c}\text { Giant }^{\mathrm{b}} \\
I_{\mathrm{B}} \\
(6)\end{array}$ & $\begin{array}{c}\text { Solar } \\
I_{\mathrm{B}}^{\mathrm{b}} \\
(7)\end{array}$ \\
\hline $\mathrm{HI}$. & 4861 & 32 & $107^{\mathrm{c}}$ & $90^{c}$ & $27^{\mathrm{c}}$ & $17^{\mathrm{c}}$ \\
\hline $\mathrm{He}_{\mathrm{I}}$ & 4471 & 6 & 5 & 4 & 1 & 0 \\
\hline He II $\ldots$ & 4686 & 24 & 18 & 18 & 17 & 16 \\
\hline He II & 1640 & 142 & 141 & 143 & 143 & 142 \\
\hline $\mathrm{C}$ III] & 1909 & $\leq 40$ & 19 & 18 & 15 & 6 \\
\hline C III. & 4650 & 12 & 9 & 12 & 1 & 0 \\
\hline $\mathrm{C}$ IV & 1549 & 1000 & 1000 & 1000 & 1000 & 1000 \\
\hline $\mathrm{N} \mathrm{III]}] \ldots$ & 1750 & $\leq 60$ & 11 & 2 & 16 & 1 \\
\hline $\mathrm{N}$ IV] ... & 1487 & 160: & 142 & 32 & 97 & 14 \\
\hline $\mathrm{N} v \ldots$ & 1240 & $\leq 100$ & 107 & 46 & 368 & 111 \\
\hline $\mathrm{O}$ III] $\ldots$ & 1666 & $\leq 70$ & 45 & 26 & 25 & 1 \\
\hline O IV] $\ldots$ & 1402 & 316 & 192 & 129 & 120 & 39 \\
\hline Si IV .... & 1397 & 316 & 6 & 3 & 4 & 0 \\
\hline O VI & 1035 & $\ldots$ & 11 & 13 & 760 & 750 \\
\hline
\end{tabular}

$$
\begin{aligned}
& { }^{\mathrm{a}} L_{\mathrm{BL}}=<3 \times 10^{33} \mathrm{ergs} \mathrm{s}^{-1} \\
& { }^{\mathrm{b}} L_{\mathrm{BL}}=2 \times 10^{35} \mathrm{ergs} \mathrm{s}^{-1} ; T_{\mathrm{BL}}=5 \times 10^{5} \mathrm{~K} . \\
& { }^{\mathrm{c}} \text { Case B prediction. }
\end{aligned}
$$

by the observed accretion disk and hard X-ray continuum alone, and models with this continuum and a boundary layer at a temperature $T_{\mathrm{BC}}=5 \times 10^{5} \mathrm{~K}$ and luminosity $L_{\mathrm{BL}}=2 \times 10^{35} \mathrm{ergs} \mathrm{s}^{-1}$. We will ignore geometric viewing angle effects (such as the $\cos i$ factor for the accretion disk luminosity) since these corrections are unknown for the hard X-ray source, and their magnitude is comparable to other uncertainties inherent to our analysis. Models with both a solar chemical composition (Lambert 1978; Lambert and Luck 1978; we assume $\mathrm{He} / \mathrm{H}=0.1$ ) and a composition appropriate for outer layers of evolved stars (Lambert and Ries 1981; Perinotto and Benvenuti 1981) are considered. This latter mixture has carbon depleted by a factor of 0.6 , nitrogen enhanced by a factor of 3 , and $\mathrm{He}, \mathrm{O}, \mathrm{Ne}, \mathrm{Mg}, \mathrm{Si}, \mathrm{S}$, and $\mathrm{Fe}$ present with solar abundances. Both mixtures are consistent with the discussion in $\S \mathrm{IV}$, although the latter may be more appropriate if the coronal gas originated in the outer envelope of the evolved late companion.

Table 7 presents the results of these calculations. The first two columns give the line identifications; the third, the reddening corrected observed intensities relative to $C$ IV $\lambda 1549$ - the intensity uncertainty is about $\pm 25 \%$ with contributions from the absolute flux calibrations, the reddening correction, and the intrinsic variation. The last four columns give the model predictions. The last two columns are models of nebulae ionized by both the observed accretion disk and $10 \mathrm{keV}$ bremsstrahlung, together with the predicted boundary layer, while the first pair of models has boundary layer emission suppressed as suggested by the Einstein observations. Tests show that these first two models would be unchanged were a boundary layer with $L_{\mathrm{BL}} \lesssim 10^{33.5} \mathrm{ergs} \mathrm{s}^{-1}$ present. The models in columns (4) and (6) have composi- tions consistent with the mean of the Lambert and Ries (1981) red giant compositions, while columns (5) and (7) have solar abundances.

The model in best agreement with observations is characterized by a red giant composition and suppressed boundary layer emission (col. [4]) and is illustrated in Figure 4. Both models with the predicted boundary layer emission have too large a $\mathrm{N} v \lambda 1240 / \mathrm{N}$ IV] $\lambda 1486$

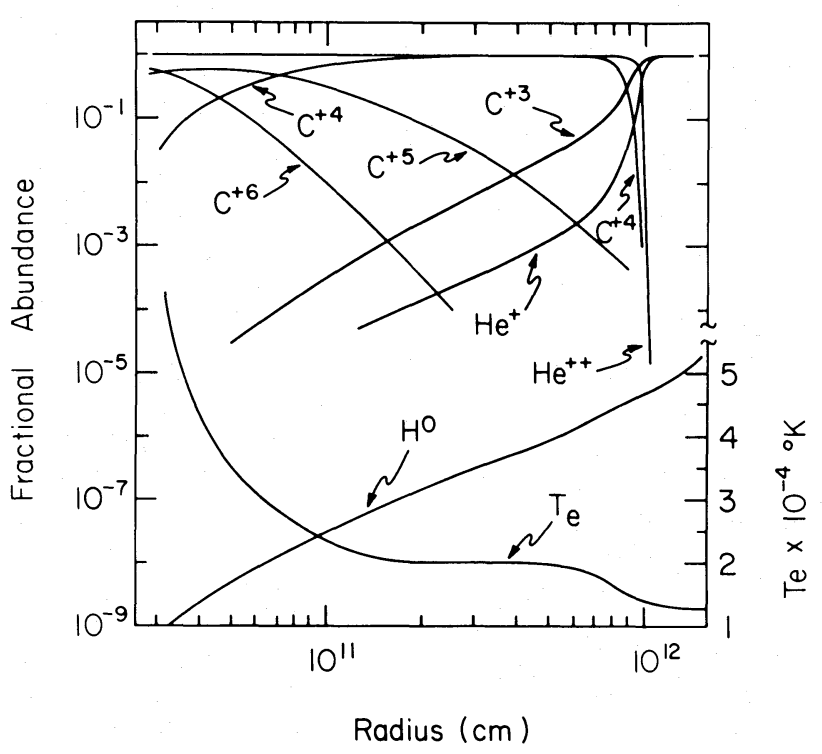

FIG. 4.-The ionization and thermal structure of the model corona with giant abundances, a constant density of $10^{10} \mathrm{~cm}^{-3}$, and an ionizing radiation field with only the observed $100 \mathrm{keV}$ bremsstrahlung emission and an extrapolated accretion disk continuum included. Distance is measured from the white dwarf. For comparison, the disk radius is $\sim 4 \times 10^{10} \mathrm{~cm}$, and the binary separation, $\sim 1.2 \times 10^{11} \mathrm{~cm}$. 
ratio, while the solar compositions have too small a $\mathrm{N}$ IV] $\lambda 1486 / \mathrm{C}$ IV $\lambda 1549$ ratio.

With the exception of the hydrogen lines, predictions are within the observational and computational uncertainties. The photoionization program does not include all the hydrogen line transfer and formation processes discussed by Drake and Ulrich (1980), and the listed intensity is a simple case $\mathrm{B}$ prediction. These results suggest that $\mathrm{H} \beta$ has been suppressed by a factor of 0.29 below case B predictions. For comparison, Drake and Ulrich found that $\mathrm{H} \beta$ was suppressed by a factor of 0.26 in their models with a similar Balmer decrement (but different photon and electron densities).

Although our model assumes specific values for the luminosity and density, tests show that the emission-line spectrum is not sensitive to exact values $( \pm 1.0 \mathrm{dex})$ of these parameters. The outer radius of the corona, which is set by the $\mathrm{C}$ IV/ $\mathrm{He}$ II ratio in our matter bounded geometry, is given by

$$
\begin{aligned}
R_{\mathrm{out}}= & 10^{12.2}\left(\frac{L}{2 \times 10^{35} \mathrm{ergs} \mathrm{s}^{-1}}\right)^{1 / 3} \\
& \times\left(\frac{N}{10^{10} \mathrm{~cm}^{-3}}\right)^{-2 / 3} \mathrm{~cm} .
\end{aligned}
$$

This large distance from the intense continuum source is needed for a gas a tenuous as $N=10^{10} \mathrm{~cm}^{-3}$ to be predominantly $\mathrm{C}^{+3}$. Emission-measure arguments show that only a small fraction of this volume can be filled with gas. An attractive model is for the coronal gas to lie in the orbital plane of the system, in which case a cylindrical geometry with radius given above and a half thickness of $10^{11.2} \mathrm{~cm}$ would be appropriate.

Córdova, Mason, and Nelson (1981) and Ferland and Truran (1981) have suggested that the observed $X$-ray continuum could be affected by the inclination of the system. Figure 5 shows the $0.1-10 \mathrm{keV}$ emergent continuum for both pole-on $\left(i=0^{\circ}\right.$, corresponding to a total column density of $\left.N_{H}=1.6 \times 10^{21} \mathrm{~cm}^{-2}\right)$ and edgeon $\left(i=90^{\circ}, N_{\mathrm{H}}=1.5 \times 10^{22} \mathrm{~cm}^{-2}\right)$ systems. These continua do not include the obscuring effects of the optically thick disk itself, of course. The coronal gas alone can completely extinguish $\nu<1 \mathrm{keV}$ radiation in edge-on systems.

Our hypothesis that the corona of V603 Aql is photoionized can be directly tested by careful observation. Both X-ray (Becker and Marshall 1981) and optical (Panek 1979) observations reveal that the continuum is highly variable over several time scales. Emission lines respond to these changes on the cooling time of the gas, estimated to be $\tau_{\text {cool }} \approx 5 N_{10}^{-1} \mathrm{~s}$ in $\S \mathrm{IV} b$, while the ionization structure changes on the recombination time scale, $\tau_{\text {rec }} \approx 1 / \alpha_{\mathrm{A}}\left(T_{e}\right) N_{e} \approx 4 N_{10}^{-1}$ minutes. For continuum variations with time scales longer than $\sim 4$

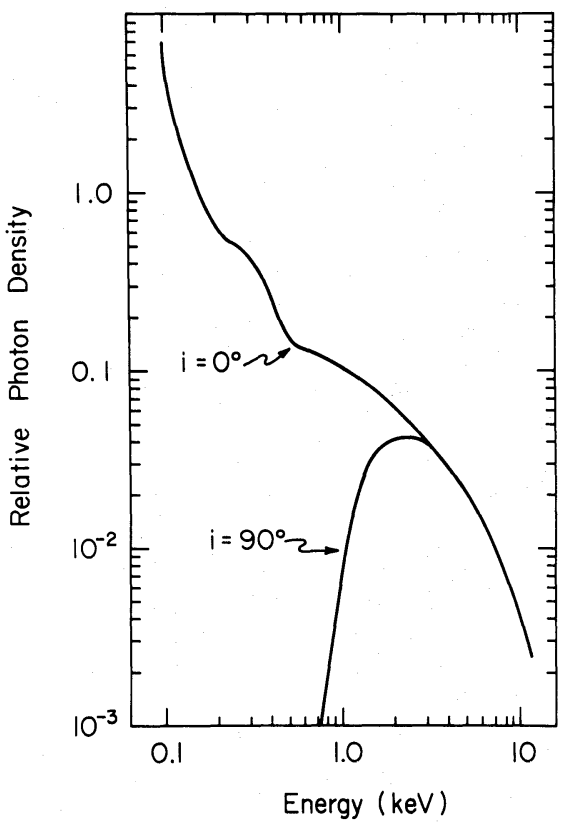

FIG. 5.-The constraints set by the observed gas density, level of ionization, and ionizing radiation field suggest that the coronal gas is a disk with radius $\sim 10^{12.2} \mathrm{~cm}$ but a half thickness of $\sim 10^{11.2} \mathrm{~cm}$. This figure compares the soft X-ray spectrum which would be observed in a pole-on system with the spectrum of an edge-on system. This assumes that all sources of radiation lie at the center of symmetry of the system and shows that the coronal gas can remove $h \nu<1 \mathrm{keV}$ radiation for $i=90^{\circ}$.

minutes, the gas will remain in equilibrium with the radiation field, steady state calculations similar to those presented above are applicable, and the effects of such changes can be calculated.

Table 8 presents predictions of the effects produced by a factor of 2 change in both the accretion disk and hard X-ray continua. The model is the best fitting model described above, and we assume that the outer radius of the corona (which sets the intensities of several emission lines) is constant and that the continuum variation is gray (the same at all frequencies). All emission-line fluxes have been scaled down by the factor appropriate for a cylindrical corona with height $h=10^{11.2} \mathrm{~cm}$, and the $\mathrm{H} \beta$ flux has been further reduced by the efficiency factor of 0.3 suggested above.

The most sensitive discriminator between our model and others will be line ratios such as $\mathrm{He}$ II $\lambda 4686 / \mathrm{H} \beta$ or He II $\lambda 1640 / C$ IV $\lambda 1549$. The nebula is optically thick to $\nu>4$ ryd photons and the $\mathrm{He}^{++}$Strömgren sphere will expand and shrink to follow continuum changes. As a result, the equivalent width of $\mathrm{He}$ II recombination lines will be constant. This contrasts with lines such as $\mathrm{H} \beta$ or C IV $\lambda 1549$ which are formed over nearly the entire nebula. In this latter case, line fluxes are set by the emission measure and will be constant. As a result ratios of lines formed in radiation-bounded zones to 
TABLE 8

EMISSION-LiNe VARIATIONS

\begin{tabular}{|c|c|c|c|}
\hline $\begin{array}{l}L_{\text {disk }} \cdots \cdots \cdots \cdots \\
L_{\mathrm{X} \text {-ray }} \cdots \cdots \cdots \\
L(1549) \ldots \ldots\end{array}$ & $\begin{array}{c}10^{35} \mathrm{ergs} \mathrm{s}^{-1} \\
10^{32.2} \mathrm{ergs} \mathrm{s}^{-1} \\
2.91 \times 10^{32} \mathrm{ergs} \mathrm{s}^{-1}\end{array}$ & $\begin{array}{c}10^{35.3} \text { ergs s}^{-1} \\
10^{32.5} \text { ergs s}^{-1} \\
3.10 \times 10^{32} \text { ergs s}^{-1}\end{array}$ & $\begin{array}{c}10^{35.6} \text { ergs s}^{-1} \\
10^{32.8} \text { ergs s}^{-1} \\
2.95 \times 10^{32} \text { ergs s}^{-1}\end{array}$ \\
\hline $\mathrm{H} \beta$ & 36 & 34 & 34 \\
\hline He I $\lambda 4471 \ldots$ & 5 & 5 & 4 \\
\hline He in $\lambda 1640 \ldots$ & 71 & 142 & 278 \\
\hline C IV $\lambda 1549 \ldots$ & 1000 & 1000 & 1000 \\
\hline $\mathrm{N}$ iv] $\lambda 1487 \ldots$ & 124 & 142 & 197 \\
\hline O IV] $\lambda 1402 \ldots$ & 43 & 64 & 98 \\
\hline
\end{tabular}

matter-bounded zones (e.g., $\mathrm{He}$ II $\lambda 4686 / \mathrm{H} \beta$ ) will vary nearly linearly with the continuum. Unfortunately, neither the continuum variations we have apparently detected nor the signal-to-noise ratio in the present data are large enough to test these predictions.

\section{CONCLUSIONS}

A simple model explains in a quantitative way several important features of the ultraviolet-optical spectrum of V603 Aql. The flat $\left(f_{v} \sim\right.$ constant $)$ continuum comes from an optically thick accretion disk. In this respect, V603 Aql is virtually identical to a dwarf nova (Bath, Pringle, and Whelan 1980). Mass transfer must proceed at a rate in $\sim 10^{18} \mathrm{~g} \mathrm{~s}^{-1}$ to support the observe luminosity of $L \geq 4 \times 10^{34} \mathrm{ergs} \mathrm{s}^{-1}$. Detailed calculations (Williams 1980) show that such an accretion disk will be optically thick throughout so that emission lines will not be formed within the disk itself.

The emission lines are probably formed in a large warm corona with dimensions comparable to the binary separation. The large radius is demanded by the emission measure of the gas and the presence of intercombination lines, notably $\mathrm{N}$ IV] $\lambda 1486$. A low temperature as deduced from the carbon emission spectrum suggest that the gas is photoionized rather than collisionally ionized. Conclusions similar to these have already been reached by Jameson, King, and Sherrington (1980) in their analysis of the dwarf nova AE Aqr.

Model calculations are presented which show that the extrapolated radiation field of the disk, together with the low luminosity X-ray continuum observed by Einstein, can maintain the heating and ionization of this gas. The chemical composition of the coronal gas is largely solar, although a mixture with depleted carbon and enhanced nitrogen gives better agreement with observations and may be more appropriate if the gas originated in the outer layer of the evolved companion. Both our calculations and the Einstein observation suggest that boundary layer emission has been suppressed in V603 Aq1.

Although we have not attempted to model its effects, the radiation emitted by the hot spot will contribute to the heating of the outer regions of the corona. Since the hot spot maintains a constant position relative to the line of symmetry between the orbiting stars, this region will follow the motion of the binary and produce the portion of the emission line undergoing the s-wave distortion.

Several features of our model are open to direct observational test. Accurate line profile analysis, particularly of the strong ultraviolet cooling lines, should help determine whether our picture is correct. Lines such as the Balmer and He II lines should be produced across much of the corona and will result in a broad line with a filled-in center. Lines such as C IV $\lambda 1549$ will be produced only across an annulus and will appear as a narrower saddle-shaped feature. A second test dealing with the effects of variability on the relative intensities of certain lines has already been outlined in the last section.

One of the goals of this study has been to investigate the differences and similarities between this old nova and the structurally similar dwarf novae. Two differences are well established (Greenstein 1960); the old novae are both brighter and show stronger He II 4686 emission. Since the brightness of the system is largely determined by the mass transfer rate, classical novae must transfer mass at a rate $\sim 10-10^{2}$ times faster than dwarf novae. If the coronal gas is indeed powered by the radiation field from the disk, then the larger mass transfer rate will produce both a harder and more luminous radiation field and hence a higher degree of ionization in the corona. In this picture, dwarf novae would have weaker $\mathrm{He}$ II 4686 because of a paucity of $\mathrm{He}^{+}$ionizing photons. Calculations (Hummer and Seaton 1964) show that $T_{\text {disk }}<5 \times 10^{4}$ would be required to entirely suppress He II 4686 in a radiation bounded geometry. The mass transfer rate required to achieve this low temperature, $\dot{m} \lesssim 10^{16.8} \mathrm{~g} \mathrm{~s}^{-1}$, is in fact consistent with the brightness of typical dwarf novae.

Many of the ideas presented in this paper were developed in conversations with J. Pringle, J. Truran, J. Whelan, and R. Williams. This research has been sup- 
ported in part by the National Aeronautics and Space Administration (grant NSG 6379), and by the National
Science Foundation through grants AST 79-22014 and 80-25222.

\section{REFERENCES}

Aldrovandi, S., and Pequignot, D. 1973, Astr. Ap., 25, 137.

Aller, L. 1956, Gaseous Nebulae (New York: John Wiley and Sons). Auer, L. 1968, Ap. J., 153, 783.

Avery, L., and House, L. 1968, Ap. J., 152, 493.

Bath, G., Evans, W., Papaloizou, J., and Pringle, J. 1974, M.N.R.A.S., 169, 447

Bath, G., Pringle, J., and Whelan, J. 1980, M.N.R.A.S., 190, 185. Becker, R. H., and Marshall, F. E. 1981, Ap. J. (Letters), 244, L93.

Bohlin, R., and Snijders, M., 1978, Memo VI to Guest Investigators, US IUE Newsletter No. 2.

Boyarchuk, A., and Mustel, E. 1964, Sov. Astr.-AJ, 8, 466.

Brocklehurst, M. 1971, M.N.R.A.S., 153, 471. 1972, M.N.R.A.S., 157, 211.

Burgess, A., and Summers, H., 1976, M.N.R.A.S., 174, 345

Córdova, F. A., Mason, K. O., and Nelson, J. E., 1981, Ap. J., 245 609.

Davidson, K., and Netzer, H., 1979, Rev. Mod. Phys., 51, 715.

Drake, S., and Ulrich, R., 1980, Ap. J. Suppl., 42, 351.

Dreschsel, H., Rahe, J., Holm, A., and Krautter, J. 1981, preprint.

Feldman, F., and MacAlpine, G. 1978, Ap. J., 221, 486.

Ferland, G. J. 1980, M.N.R.A.S., 191, 243.

Ferland, G. J., and Truran, J. 1981, Ap. J., 244, 1022.

Gallagher, J., and Holm, A. 1974, Ap. J. (Letters), 189, L123.

Greenstein, J. 1960, in Stars and Stellar Systems, Vol. 6, Stellar Atmospheres, ed. J. Greenstein (Chicago: University of Chicago Press), p. 676.

Halpern, J. P., and Grindlay, J. E. 1980, Ap. J., 242, 1041.

Harrington, J., Lutz, J., Seaton, M., and Stickland, D. 1980, M.N.R.A.S., 191, 13 .

Hayes, D., and Latham, D. 1975, Ap. J., 197, 593.

Henry, R. J. W. 1982, private communication.

Hummer, D., and Seaton M. 1964, M.N.R.A.S., 127, 217.

Jameson, R., King, A., and Sherrington, M. 1980, M.N.R.A.S., 191, 559.

Kiplinger, A. 1978, Ph.D. thesis, University of Texas.

. 1979, Ap.J., 234, 997.

Kraft, R. 1964, Ap.J., 139, 457

Krolik, J., and McKee, C. 1978, Ap. J. Suppl., 37, 459.

Lambert, D. L. 1978, M.N.R.A.S., 182, 249.

Lambert, D. L., and Luck, R. E. 1978, M.N.R.A.S., 183, 79.

Lambert, D. L., and Ries, L. 1981, Ap. J., 248, 228.

Lambert, D. L., Slovak, M. H., Shields, G. A., and Ferland, G. J. 1980, in The Universe at Ultraviolet Wavelengths, NASA Conf. Pub. 2171, p. 546.
Lynden-Bell, D. 1969, Nature, 223, 690.

McLaughlin, D. B. 1960, in Stars and Stellar Systems, Vol. 6, Stellar Atmospheres, ed. J. Greenstein (Chicago: University of Chicago Press), p. 585.

Nandy, K., Thompson, G., Jamar, C., Monfils, A., and Wilson, R., 1976, Astr. Ap., 51, 63.

Netzer, H. 1975, M.N.R.A.S., 171, 395. 1977, M.N.R.A.S., 178, 489

Nussbaumer, H., and Storey, P. 1975, Astr. Ap., 44, 321.

Osterbrock, D. 1974, Astrophysics of Gaseous Nebulae (San Francisco: Freeman)

Panek, R. 1979, Ap. J., 234, 1016

Payne-Gaposchkin, C. 1957, The Galactic Novae (Amsterdam: North-Holland)

Pengelly, R. 1963, Ph.D. thesis, University College London.

Perinotto, M., and Benvenuti, P. 1981, Astr.Ap., 100, 88.

Pringle, J. E. 1977, M.N.R.A.S., 178, 195.

Pringle, J., and Savonije, G. 1979, M.N.R.A.S., 187, 777.

Raymond, J., Cox, D., and Smith, B. 1976, Ap. J., 204, 290.

Robinson, E. 1976, Ann. Rev. Astr. Ap., 14, 119.

Seaton, M. J., $1978 a, M . N . R . A . S ., 185,5 p$. $1978 b$, in IAU Symposium 76, Planetary Nebulae, Observations and Theory, ed. Y. Terzinn (Dordrecht: Reidel), p. 131 .

Shuder, J. M., and MacAlpine, G. M. 1979, Ap. J., 230, 348.

Slovak, M., 1981, Ap. J., 248, 1059.

Stockman, H., Schmidt, G., Angel, R. P., Leibert, J., Tapia, S., and Beaver, E. 1977, Ap. J., 217, 815.

Storey, P. 1981, M.N.R.A.S., 195, 27 P.

Tylenda, R. 1977, Acta Astr., 27, 235.

Warner, B., 1976, in IAU Symposium 73, The Structure and Evolution of Close Binary Systems, ed. P. Eggleton, S. Mitton, and J. Whelan (Dordrecht: Reidel), p. 85.

Warner, B., and Nather, R. 1971, M.N.R.A.S., 152, 219.

Weymann, R., and Williams, R. 1969, Ap. J., 157, 1201.

Williams, R. E. 1980, Ap. J., 235, 939

Wills, D., and Netzer, H. 1979, Ap. J., 233, 1

Wyckoff, S., and Wehinger, P., 1978, in IAU Colloquium 42, The Interaction of Variable Stars with their Environment, ed. R. Kippenhahn, Veröff. Remeis-Sternw. Bamberg, 121, 201.

\section{G. J. FeRLAND: Department of Physics and Astronomy, University of Kentucky, Lexington, KY 40506}

D. L. Lambert, M. L. McCall, G. A. Shields, and M. H. SlovaK: Department of Astronomy, University of Texas, Austin, TX, 78612. 